\title{
The knee magnetic resonance order: can it be reduced by specialists?
}

\begin{abstract}
Introduction

Knee pain is one of the most common complications in the trauma clinic. It is common to find patients who come to the office with a Nuclear Magnetic Resonance (MRI) who have a broken meniscus and who are looking for a lathe in the operating room. The majority of these patients are feasible for orthopedic treatment and it is sufficient to perform correct semiological maneuvers to determine it. Although there is a growing demand for knee MRI by members of the health team. The hypothesis of this work postulates that the NMR requested by the specialists in orthopedics and traumatology offer positive results both for the diagnoses and for the treatment plans.
\end{abstract}

Objectives: The aim of this study is to compare the usefulness in diagnosis and in the therapeutic definition of knee MRIs requested by medical specialists.

Materials and methods: We retrospectively reviewed 1,186 knee MRI reports from January 1, 2015 to July 31, 2015, using the computerized clinical history system. These were classified according to the origin of the request for the images (specialists in orthopedics and traumatology, residents of traumatology and other medical specialties). This information was explored for its characterization.

Retrospective study. Level of evidence IV

Results: We obtained 33\% (389) normal report of knee, significantly lower $(\mathrm{p}<0.001)$ to $67 \%$ reporting injuries.

The proportion of studies requested was mostly by specialists in orthopedics and traumatology (59\%), followed by the proportion required by different medical specialties $(27 \%)$ and by those prescribed by traumatology residents (13\%). The percentages of normal reports of those studies requested were different $(p<0.05) 29,42$ and $32 \%$, respectively.

Conclusion: In primary care by physicians and residents of traumatology, the request for knee MRI reports a high percentage of normal reports. Specialists in orthopedics and traumatology request this exam with greater demand and present greater findings of injuries.

Keywords: Knee RMI; Knee injuries; Medical order
Volume 10 Issue 3 - 2018

\author{
Damian Gabriel Bustos, Ivan Bitar, Lucas M \\ Arangoni, Pablo Bertiche, Cesar Orellana \\ Cordoba, University Universidad NAcional de Cordoba, \\ Argentina
}

\section{Correspondence: Damian Gabriel Bustos, Cordoba, University Universidad NAcional de Cordoba, Ana Maria janer 1067, Argentina, Tel 0I I-35I2248I94,}

Email damian5butstos@hotmail.com

Received: April 20, 2018 | Published: June 21, 2018

\section{Introduction}

Knee pain is one of the most common consultations in the trauma clinic. In the number of annual consultations in the United States during the period 1995-1996 according to the CSC (Center for Disease Control and Prevention), there were more than 16 million and approximately 5.7 million of these consultations were made to specialists in orthopedics and traumatology, knee pain being the most frequent consultation in the office, almost twice as much as back pain. ${ }^{1}$ It is common to find patients who come to the consultation with a Nuclear Magnetic Resonance (MRI) saying that they have a broken meniscus and looking for a shift in the operating room. The majority of these patients are feasible for orthopedic treatment and it is sufficient to perform correct semiological maneuvers to determine it. Despite this, there is a growing number of requests for MRI of the knee by members of the health team. In adults with gonalgia, the physical examination and the anamnesis in the consultation is usually sufficient. If the patient does not report a history of knee entorsis followed by joint swelling and functional impotence, it is difficult to have a ligament rupture. This allows the doctor to distinguish between pathologies that may require additional images and probable surgical treatment and conditions that are amenable to non-surgical treatment. ${ }^{2}$
Magnetic Resonance Imaging (MRI) is effective for the identification of meniscal, ligament, bone, and neurovascular lesions, among others ${ }^{3}$ being of great value in multiligamentary lesions, for example, but always accompanied by a physical examination, as Claude's saying goes Bernard, who does not know what he is looking for, does not know what he finds. However, this study is costly, time consuming, with a marked tendency towards an excess of findings or erroneous diagnoses. Often, MRI is considered unnecessary for most outpatients with initial complaints of knee pain if a trauma and / or previous entorsis. ${ }^{4}$ Besides the high economic cost in the health system, in many occasions it can lead to an inappropriate treatment for the patient. Inappropriate use of MRI can distort patient expectations, offer false positive or incidental reports, condition to continue unnecessary consultations with the knee specialist and can lead to unnecessary and ultimately ineffective surgical procedures. ${ }^{5}$ It is probable that if we perform MRI of the knee in elderly patients we will find meniscal lesions that are also probably degenerative and are not the reason for the pain. The objective of this work is to describe the usefulness of the MRI of knees requested and compare their prescriptions for different medical specialties. Our hypothesis postulates that the NMR requested at the Sanatorium Allende by specialists in orthopedics and traumatology have greater positive results both for the diagnoses and for the treatment plans. 


\section{Materials and methods}

A retrospective study was carried out at the Sanatorium Allende (Córdoba), from January 1, 2015 to July 31, 2015. Using the computerized medical records system, an exhaustive search was carried out and information was collected from the knee MRI reports. The inclusion criteria for the selection were: subjects of both sexes and those older than 15 years who did not have a history of fractures, previous surgeries. And as exclusion criteria, the presence of an NMR report pending or indicated by a doctor not belonging to the Sanatorium Allende. 1186 reports were obtained. The MRIs were classified and organized on a basis, according to the medical specialty of the requesting physician, specifying the sex and age of the patient, results of the MRI report and its date, knee studied, data referring to the condition, social work, particular or with an occupational risk insurer (ART) and according to the treatment indicated after the MRI report (surgical-medical). An exploratory statistical analysis (measurements, summary and descriptive graphs) was carried out initially, and tests of independence in contingency tables, by Chi-square test, for the identification of associations between variables.

\section{Results}

We obtained 1186 reports of MRI of knees, being 32.8\% (389) normal and $67.20 \%$ with lesions ( $\mathrm{P}<0.001)$. For the statistical analysis we used the stata V 14 software (Stata Coorp, texas, USA). The mean age of the patients was $36( \pm 14)$ years, being the extreme ages 76 and 15 years and the studies were carried out, mostly in the male sex $(67 \%$, 797). The largest number of reports corresponded to trauma physicians (specialist) (59\%) followed by professionals from other specialties $(27 \%)$ and in their minority by traumatology residents (13\%) (Figure 1). The NMR reports from the requests by orthopedic surgeons were about $29 \%$ normal, with the lesions found, mostly, characterized by internal meniscal rupture $(18 \%)$, followed by osteochondritis and internal meniscal rupture $9 \%$. Regarding physicians from other specialties, $42 \%$ of the MRI reports were normal. The lesions found in this category were also characterized by internal meniscal rupture $(17 \%)$ and ACL rupture - osteochondritis. $32 \%$ of the MRI reports prescribed by trauma residents were normal and the lesions reported were ACL rupture (13\%), followed by rupture of the internal meniscus and osteochondritis $(12 \%-12 \%)$. The treatment established, based on the MRI reports, was mainly unincumbered in $86 \%$ of the cases and only $14 \%$ of the patients received some kind of surgical treatment in the institution. This distribution was not associated to the category of the professional (doctors of other specialties, traumatologists or residents of traumatology, $\mathrm{P}=0.354$ ), nor to the age of the patients $(\mathrm{p}=0.142)$.

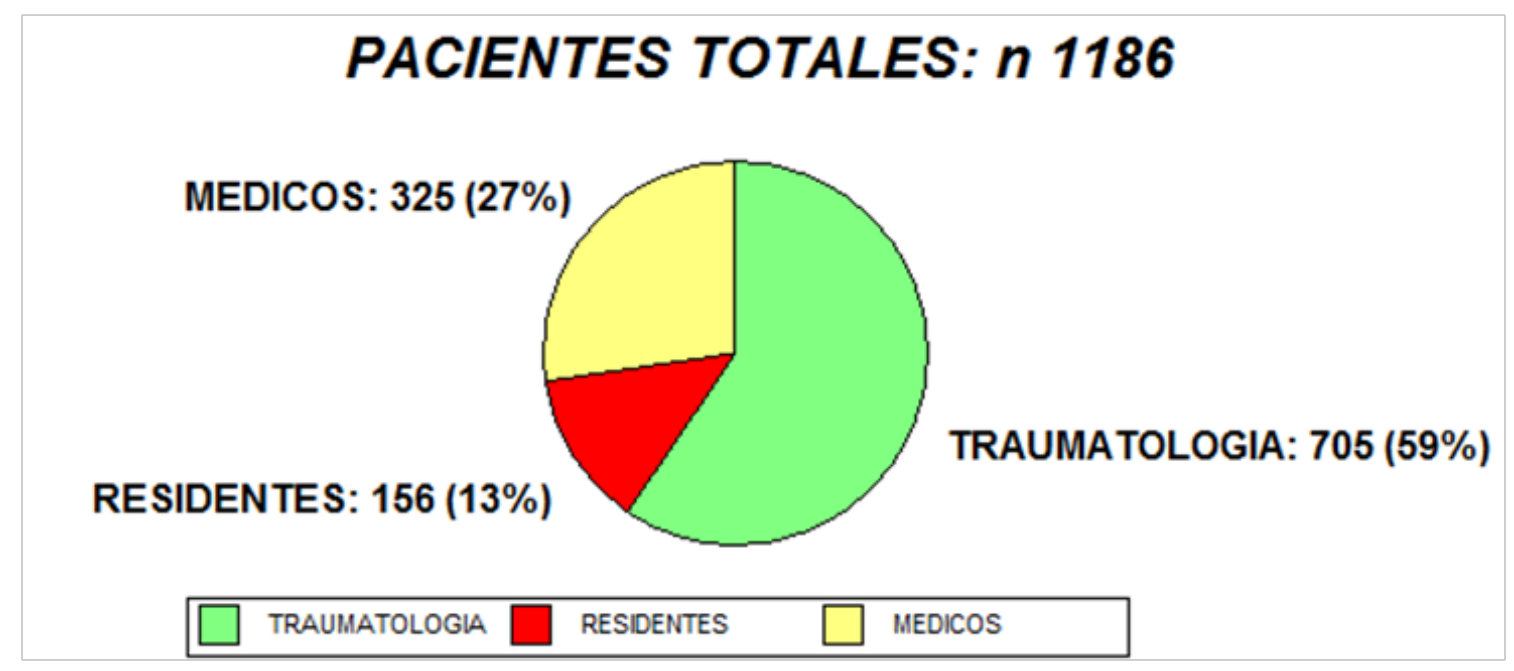

Figure I Proportion of NMR reports requested by the categories of medical professionals. Sanatorium allende, January-July 2015.

\section{Discussion}

The progress of Medical Sciences and Technology has led to the increase of complementary requests for diagnostic imaging, including the procedures of non-invasive techniques such as Magnetic Resonance Imaging (MRI), computerized axial tomography (CAT). There is a clear relationship between the availability of technology and the use that is generated. The Sanatorium Allende is a center of high complexity and the NMR studies of knees performed in that institution were numerous and the majority requested by specialists in traumatology and orthopedics, having a considerable percentage of reports of injuries. Solomon et al. in a retrospective study it was shown that the knee MRI number was doubled, with fewer studies requested by trauma specialists. ${ }^{6}$ There is a wide range of factors that influence the excessive use of MRI, such as financial incentives, defensive medicine and patient demand. ${ }^{7}$ Sherman et al. have demonstrated that specialists in orthopedics and traumatology are more inclined to request MRI, indicating more frequently in patients with clinically important lesions in the menisci, cartilage and cruciate ligaments. ${ }^{8}$ Petron et al. define that MRI can detect degenerative changes without clinical significance and that they are related to age, and these results may confuse the diagnosis and treatment plans in primary care physicians and that $17 \%$ of the total studies requested by physicians from Primary care would eventually have changes in the treatment plan by the trauma specialist. ${ }^{9}$ Although the retrospective design of this study can be considered as a limitation, there is a clear difference in the results of requests for magnetic resonance of the knee, by specialists in orthopedics and traumatology or residents of traumatology and different medical specialties. This is demonstrated by the studies of recent years. Roberts et al. They analyzed patients 
divided into two groups: those who were initially evaluated by primary care physicians and those initially evaluated by trauma specialists. In NMRs ordered by traumatology specialists, a large number of injuries were diagnosed and greater surgical interventions were performed compared to those requested by primary care physicians. ${ }^{5}$

\section{Conclusion}

Our data conclude that the request for MRI of the knee in primary care by doctors and residents of traumatology have a greater number of normal reports. The specialists in orthopedics and traumatology request with greater demand and present greater findings of injuries.

\section{Acknowledgements}

None.

\section{Conflict of interest}

The auhtor declares there is no conflict of interest.

\section{References}

1. Susan M. Schappert MA. Office Visits to Orthopedic Surgeons: United States, 1995-96 by, Division of Health Care Statistics. 1998. p. 1-31.

2. Quatman CE, Hettrich CM, Schmitt LC, et al. The clinical utility and diagnostic performance of magnetic resonance imaging for identification of early and advanced knee osteoarthritis: a systematic review. Am J Sports Med. 2011;39(7):1557-1568.
3. Kumaravel M, Weathers WM. Emergency Magnetic Resonance Imaging of Musculoskeletal Trauma. Magn Reson Imaging Clin N Am. 2016;24(2):391-402.

4. Muyibat A, Adelani MD, Nathan A. et al. The Use of MRI in Evaluating Knee Pain in Patients Aged 40 Years and Older. J Am Acad Orthop Surg. 2016;24(9):653-659.

5. Roberts TT, Singer N, Hushmendy S, et al. MRI for the evaluation of knee pain: comparison of ordering practices of primary care physicians and orthopaedic surgeons. J Bone Joint Surg Am. 2015;97(9):709-714.

6. Solomon DH, Katz JN, Carrino JA. Trends in knee magnetic resonance imaging. J Med Care. 2003.

7. Oikarinen H, Karttunen A, Paakko E, et al. Survey of inappropriate use of magnetic resonance imaging. Insights Imaging. 2013;4(5):729-733.

8. Sherman PM, Penrod BJ, Lane MJ, et al. Comparison of knee magnetic resonance imaging findings in patients referred by orthopaedic surgeons versus nonorthopaedic practitioners. Arthroscopy. 2002;18(2):201-205.

9. Petron DJ, Greis PE, Aoki SK, et al. Use of knee magnetic resonance imaging by primary care physicians in patients aged 40 years and older. Sports Health. 2010;2(5):385-390. 